Estudios Románicos, Volumen 28, 2019, pp. 361-373

ISSN: 0210-491

eISSN: 1989-614X

DOI: https://doi.org/10.6018/ER/373771

\title{
"SINGULAR DECUS YTALICUM": LA BIOGRAFIA DI GIOVANNA DI NAPOLI NEL DE MULIERIBUS CLARIS
}

("Singular decus ytalicum": the biography of Joanna of Naples in the De mulieribus claris)

Francisco José Rodríguez Mesa*

Universidad de Córdoba

\begin{abstract}
The last of the one hundred and six chapters conforming Boccaccio's De mulieribus claris is devoted to queen Joanna of Naples. The addition of this life implies a certain degree of anomaly in the tradition of exemplary literature because of two reasons. Firstly, the Angevin sovereign was in her early thirties when the author finished the composition of his collection and, secondly, because the description of the monarch's life that Boccaccio provides can be said far from objective narration and much closer to a propagandistic portrait of the Neapolitan queen.

In this article, these particularities of Joanna's life are analyzed at the time that the reasons that could lead Boccaccio to configure in such a way the concluding chapter of her anthology of famous women are examined.
\end{abstract}

Keywords: Boccaccio; De mulieribus claris; Exemplary women tradition; Joanna of Anjou; Angevine Naples; Women in Medieval literature.

Riassunto: L'ultimo dei centosei capitoli del De mulieribus claris di Boccaccio è consacrato alla regina Giovanna di Napoli. Quest'inclusione comporta un'anomalia nella topica della letteratura esemplare per due motivi. Per cominciare, la sovrana angioina era poco più che trentenne quando il certaldese finì la stesura della sua silloge e, in secondo luogo, perché la versione della vita della monarca che l'autore fornisce è ben lungi da poter essere considerata una narrazione oggettiva, avvicinandosi molto di più a un ritratto propagandistico della regina partenopea.

* Dirección para correspondencia: Francisco José Rodríguez Mesa. Dpto. de Ciencias del Lenguaje. Área de Filología Italiana.Facultad de Filosofía y Letras. Plaza del Cardenal Salazar s/n. 14071 Córdoba. (francisco.rodriguez.mesa@uco.es) 
In questo articolo si analizzano queste particolarità della vita di Giovanna e si indaga nei motivi che potrebbero aver portato Boccaccio a configurare in questo modo il capitolo di chiusura della sua raccolta sulle donne celebri ${ }^{1}$.

Parole chiave: Boccaccio; De mulieribus claris; letteratura esemplare femminile; Giovanna d'Angiò; Napoli angioina; donna nella letteratura medioevale.

La natura della letteratura esemplare, dal punto di vista dei suoi recettori, può dirsi quanto meno particolare. Da una parte, si tratta di un prodotto artistico la cui principale caratteristica dovrebbe essere, appunto, la bellezza letteraria o, quanto meno, il piacere che dalla lettura di essa si dovrebbe trarre. Dall'altra parte, sebbene questa qualità artistica costituisca una componente essenziale, il genere implica quella messa in atto della letteratura di cui Petrarca parlò nella sua Familiare I, III, 42; vale a dire, queste opere dovrebbero spingere il lettore a mettere in pratica le conoscenze acquisite in modo che se ne tragga un miglioramento personale. Da questa combinazione -che richiama l'oraziano docere et delectare- sorge un fenomeno straordinariamente ricco e singolare: secondo l'intenzionalità di questo genere, l'opera esemplare, pur appartenendo alla sfera letteraria, dovrà sortire una serie di effetti nel mondo reale e, quindi, extraletterario.

Per riuscire ad oltrepassare i limiti del testo letterario, gli autori di opere esemplari hanno fatto del loro meglio per assicurare ai loro testi una validità il più universale possibile, rifuggendo apertamente, evitando sottilmente o spiegando con il massimo zelo didattico tutte quelle circostanze o tutti quei personaggi con cui il lettore potesse trovarsi in disaccordo o da cui egli non potesse trarre gli opportuni insegnamenti.

Così, impeccabili sono le biografie dei centotrentacinque personaggi dei primi tempi del Cristianesimo scelti da San Girolamo, il primo coltivatore del genere della nostra era, nel cui De viris illustribus si trova il germe del recupero della tipologia testuale nel Rinascimento italiano. Altrettanto palese nella sua determinazione di vocazione universale del genere è Petrarca, la cui opera omonima a quella di Girolamo (1999) è divisa in due parti ancora più nette ma similmente indiscutibili rispetto a quelle del Padre della Chiesa.

La prima delle due sezioni del catalogo petrarchesco è formata dai personaggi dell'Antichità Classica, le cui gesta sono in parte ricavate dall' Africa, e che di solito sorgono come esempi incontestabili di valore, ardore e forza guerriera o di magnanimità e giustizia nel governo. La seconda parte dell'opera, notevolmente più breve, è occupata da personaggi biblici a cui solitamente si allude con propositi più utili alla vita morale che non all'ambito sociale o politico.

1 Questa pubblicazione si inserisce nel Progetto di Ricerca del Ministerio de Economía y Competitividad spagnolo "Voces de mujeres en la Edad Media: realidad y ficción (siglos XII-XIV)", FFI2014-55628-P.

2 In consonanza con la svolta verso la messa in pratica dell'erudizione che l'aretino sperimentò dopo aver superato i quarant'anni e di cui Rico già parlò (2002: 59-61), Petrarca scrisse nella sua Familiare I, III, 4 che lo studio deve orientarsi "magis [...] ad vitam quam [...] ad eloquentiam" (in Rico 2002: 59-61). 
Lasciando da un lato i personaggi che ne fanno parte, il De viris illustribus petrarchesco può dirsi il paradigma o l'archetipo del recupero della letteratura esemplare dopo i tempi classici, sia per la sua struttura, semplice ma chiara che, in gran parte, lascia intravedere un criterio cronologico; sia per i principi da cui sembra derivare la selezione dei personaggi raccolti.

Partendo, nel primo libro, da Romolo e arrivando a Traiano e, nella seconda parte, con un percorso che va da Adamo ad Ercole, la lontananza cronologica fra i personaggi esemplari e l'autore e il suo pubblico è una componente primordiale del testo petrarchesco. Sarebbe troppo ardito determinare i motivi per i quali l'aretino rifiuta modelli cronologicamente più vicini ${ }^{3}$, probabilmente per i principi culturali inerenti agli studia humanitatis, o forse per la facilità di abbracciare un corpus omogeneo e indiscusso di biografie.

Tuttavia, a nostro avviso, da questa scelta deriva una delle principali ragioni per le quali il De viris illustribus ebbe una straordinaria fortuna nella civiltà letteraria del rinascimento europeo e, soprattutto, questa caratteristica potrebbe spiegare le scarse modifiche che il testo petrarchesco sofferse nei suoi volgarizzamenti. Ovviamente, le biografie attinenti alla tradizione classica o biblica erano capite -quando non perfettamente note- da ogni lettore colto in ogni angolo del Vecchio Continente.

Circa all'inizio dell'estate del $1361^{4}$, Boccaccio decide di imitare il modello del $D e$ viris illustribus per il suo De mulieribus claris, e lo dice apertamente nell'incipit del proemio ${ }^{5}$ :

Scripsere iam dudum non nulli veterum sub compendio de viris illustribus libros; et nostro evo, latiori tamen volumine et accuratiori stilo, vir insignis et poeta egregius Franciscus Petrarca, preceptor noster, scribit; et digne. Nam qui, ut ceteros anteirent

3 Le parole dell'aretino all'inizio del proemio del De viris illustribus sono poco illuminanti sotto questo aspetto e fanno solo riferimento ai limiti di estensione che l'opera deve rispettare:

Quis enim, queso, Parthorum aut Macedonum, quis Gothorum et Unnorum et Vandalorum atque aliarum gentium reges ab ultimis repetitos in ordinem digerat, quorum et obscura semper et iam senio deleta sunt nomina? quod si aggrediar, ut laboris ac temporis iactura sileatur et operis immensitas et legentium fastidium, nonne propositi mei videbor oblitus? Neque enim quisquis opulentus et potens confestim simul illustris est; alterum enim fortune, alterum virtutis et glorie munus est; neque ego fortunatos sed illustres sum pollicitus viros. (Petrarca 20072012: 222)

[Chi, di grazia, potrebbe registrare in ordine, dai più lontani, i re dei Goti, degli Unni, dei Vandali e d'altre genti, i cui nomi, oscuri sempre, sono ora obliterati dal tempo? Se mi provassi a farlo, a tacere della perdita di tempo e di fatica, dell'immensità dell'opera e del fastidio dei lettori, non sembrerei dimentico del mio proposito? Non ogni ricco è potente e per ciò stesso illustre: l'una cosa e dono di fortuna, l'altra di virtù e di gloria: né io vi ho promesso uomini fortunati, ma illustri.] (Petrarca 2007-2012: 223)

$4 \quad$ È da decenni che le fasi di composizione e, dunque, la datazione del $D M C$ sono state stabilite con una chiarezza che ammette pochi dubbi. Nel primo studio che pose le basi di quest'argomento, Ricci (1959: 132-134) stabilì sette fasi redazionali mentre, pochi anni dopo, Zaccaria (1963: 293) ne distinse nove. Tuttavia, le divergenze fra entrambe le posizioni - come Müller (1992: 18-21) dimostrò - non possono dirsi inconciliabili. Per ulteriori informazioni sulla datazione e le modifiche che la silloge presenta nei manoscritti che la tramandano, vedi anche Zappacosta (1973), Branca-Zaccaria (1996) e Nuvoloni (2003).

5 Bisogna ricordare che l'ispirazione petrarchesca nelle opere del certaldese non si limita al $D M C$, ma si cita anche in modo esplicito nell'altro compendio di vite del Boccaccio latino, il De casibus virorum illustrium (VIII, I, 2-6). Per approfondire nel rapporto fra entrambi gli autori, vedi Rico (2013) e Roush (2015). 
claris facinoribus, studium omne substantias, sanguinem et animam, exigente oportunitate, posuere, profecto ut eorum nomen in posteros perpetua deducatur memoria mervere $^{6}$. (Boccaccio 1967: 22-24)

La raccolta del certaldese è di capitale importanza perché, eccezione fatta per il $D e$ mulierum virtutibus di Plutarco e per dirlo con le parole di Filosa, "per la prima volta, ispirandosi nella struttura alle opere che trattavano degli uomini illustri, il genere encomiastico della biografia viene applicato al mondo femminile" (2012: 48) ${ }^{7}$.

All'esposizione di biografie muliebri sottostà un criterio cronologico. Così, si parte dalla prima delle donne, Eva, e tramite una serie di dee pagane e personaggi dell'Antichità si arriva ad un'ultima sezione di sei capitoli che contiene le vite di altrettanti personaggi di epoca post-classica fino alla chiusura con la regina Giovanna di Napoli.

In questo studio focalizzeremo la nostra attenzione su quest'ultima sezione dell'opera. In essa, come in tutto il resto della silloge, si può costatare quella propensione che Boccaccio sviluppò durante il periodo napoletano consistente nel raccontare le vite di sovrani e che può anche osservarsi nel De casibus (Billanovich 1953). Ciononostante, questa divisione spicca per certe disimmetrie con la tradizione dei compendi di biografie esemplari per diversi motivi.

La parte conclusiva della raccolta è composta dalle vite di:

- Giovanna anglica, papessa (CI)

- Irene, imperatrice di Costantinopoli (CII)

- Gualdrada, vergine fiorentina (CIII)

- Costanza, imperatrice romana e regina di Sicilia (CIV)

- Camiola, vedova senese (CV)

- Giovanna, regina di Gerusalemme e di Sicilia (CVI)

Come conseguenza del rispetto del criterio cronologico dell'esposizione precedentemente sottolineato, la difficoltà di estrapolare gli insegnamenti dalle vite di quest'ultima parte dell'opera aumenta man mano il lettore si avvicina alla fine della silloge. La narrazione di Gualdrada potrebbe dirsi un esempio universale di difesa della castità femminile contro la minaccia di un potere politico forte e quella di Costanza rimanda ad una vicenda storica che, nonostante non sia ampiamente nota, sarebbe stata facile da capire da parte di lettori non italiani grazie all'enfasi didattica del Boccaccio. Ciononostante, gli ultimi due capitoli sembrano di essere il frutto di un approccio ben diverso e la struttura stessa delle biografie ne evidenzia alcune tracce. Così, la vera e propria

$6 \quad$ Offriamo per ogni citazione latina del $D M C$ la traduzione di Zaccaria nell'edizione utilizzata.

Già nel passato alcuni degli antichi storici scrissero compendi intorno agli uomini illustri. Ai nostri tempi uno di maggior estensione e più accurato stile lo sta scrivendo il mio maestro Francesco Petrarca, uomo insigne ed egregio poeta. E ben a ragione: poiché meritarono che il loro nome fosse consegnato a perenne ricordo ai posteri, quegli uomini che, per superare gli altri in azioni egregie, spesero tutte le loro forze e le loro sostanze; e, se fu necessario, donarono anche il sangue e la vita. (Boccaccio 1967: 21-23)

7 Questo carattere innovativo del Boccaccio rispetto il $D M C$ fu già evidenziato dai Hortis (1877) e Torretta (1902a; 1902b), primi studiosi moderni della silloge. 
storia di Camiola non è narrata che dopo un lungo excursus storico che riguarda fatti attinenti quasi esclusivamente alla storia italiana e con una rilevanza scarsa -o perfino un interesse nullo- in territori d'Oltralpe. Ancora di più colpisce l'inclusione della regina Giovanna, unico personaggio vivo tra tutti quelli della raccolta e del quale, da un punto di vista completamente oggettivo, non si racconta nessuna gesta concreta.

In altri termini, il tratto più immediato e palese di questa sezione, tenuto conto delle caratteristiche precedentemente esposte, è che, lasciando da parte i capitoli contenenti le storie della papessa Giovanna e dell'imperatrice Irene, le altre donne appartengono sia da un punto di vista storico, sia da una prospettiva geografica, ad ambienti particolarmente vicini all'autore e al suo pubblico più immediato. Dunque, l'approccio universalista che caratterizzò la raccolta maschile del Petrarca è estraneo alla chiusura del $D M C$.

Visto questo ingrediente fortemente indigeno della sezione finale non c'è da meravigliarsi se, al contrario di quanto si affermava rispetto all'opera dell'aretino, la conclusione della collezione di biografie muliebri sofferse non poche variazioni nel corso dei suoi adattamenti a culture non italiane tramite processi di traduzione. In effetti, per esempio, la prima traduzione in spagnolo, De las ilustres mujeres en romance (Bocacio 1494), edita a Saragozza nel 1494, elimina le ultime due biografie ${ }^{8}$, mentre la prima traduzione in tedesco, Von etlichen Frowen, fatta da Heinrich Steinhöwel e pubblicata a Ulm nel 1473, sopprime anche i capitoli CV e CVI e si chiude con la traduzione in tedesco della Griselda decameroniana tramite la versione petrarchesca contenente nel De insigni obedientia et fide uxoria9.

Per approfondire l'analisi di questa sezione vale la pena di soffermarsi sulla struttura delle biografie che la compongono. È da decenni che gli studiosi del compendio muliebre osservano che

I capitoli rivelano, spesso, una comune sottostruttura interna. La gran parte delle biografie inizia, infatti, con un primo paragrafo dedicato a un inquadramento storico, geografico e sociale della protagonista: il narratore informa il lettore sul periodo e sui luoghi in cui visse la donna, e sulla sua famiglia. Solo successivamente si entra nel vivo della biografia: vengono delineate le caratteristiche fisiche e umane della protagonista (bellezza, intelligenza, abilità...) e le azioni che l'hanno resa celebre. In molti casi il capitolo termina con un'esortazione, considerazioni di vario genere o con inserti moraleggianti, non di rado svincolati dall'episodio appena letto. (Filosa

\footnotetext{
8 Questa traduzione fu pubblicata in edizione facsimile dalla Real Academia Española (Boccaccio 1951) ed è stata studiata da Fernández Murga e Pascual Rodríguez (1975; 1977) e da Díaz-Corralejo (2001). Come quest'ultima studiosa sottolinea, la versione spagnola sembra prendere come testo di partenza il manoscritto 10.000 della Biblioteca Nacional de España, nel quale mancano anche le vite di Camiola e di Giovanna (Díaz-Corralejo 2001: 240-241). Queste mancanze potrebbero anche evidenziare che il suddetto manoscritto non fu redatto in Italia.

9 Steinhöwel omette anche il capitolo LXXXVI, dedicato alla vita di Cornificia. Per quanto riguarda il suo modo di concludere la compilazione boccaccesca, bisogna ricordare che, oltre all'ingente fortuna di cui godette la storia di Griselda in tutta Europa dalla fine del Trecento in poi (vedi Morabito 1988; 1990; 1993 e, più di recente, Nardonne 2000; 2001), gli insegnamenti di questa narrazione erano molto più universali che quelli della conclusione originale del $D M C$. Per maggiori dettagli sulle modifiche di Steinhöwel rimandiamo a Rodríguez Mesa (2009).
} 
2012: 17)

Spesso, questa struttura risale ad altri modelli letterari in lingua vernacola. Come già notò Müller, "Aus den über 100 Frauenviten in $D M C$ ragen einzelne durch die Lebendigkeit der Darstellung und die sorgfältige literarische Gestaltung heraus. In vielen dieser Kapitel fallen novellenartige Strukturen und Themen auf, die Decameron bekannt sind" (1992: 84). Senza perdere di vista queste affermazioni, vediamo le parti in cui si possono suddividere le ultime quattro biografie dell'opera ${ }^{10}$ :

CIII, Gualdrada, vergine fiorentina:

1. $\left[1 \mathrm{a}^{11}\right]$ Genealogia

2. [1b] Motivi per cui se ne deve parlare

3. [2-7] Storia

4. [8] Utilità della storia

CIV, Costanza, imperatrice romana e regina di Sicilia

1. [1] Motivi per cui se ne deve parlare

2. [2a] Genealogia

3. [2b-9] Storia

4. [10] Riflessione sulla storia

$\mathrm{CV}$, Camiola, vedova senese

1. [1-2a] Presentazione e genealogia

2. [2b-12] Contesto storico

3. [13-32] Storia ${ }^{12}$

CVI, Giovanna, regina di Gerusalemme e di Sicilia

1. [1] Introduzione

2. [2-4] Biografia dettagliata

3. [5-7] Descrizione dei suoi possedimenti

4. [8-14] Virtù

a. [8-11] Virtù di governo e di carattere

b. [12] Bellezza e atteggiamento

c. [13-14] Conclusione

Se si contrappone alla struttura delle tre storie precedenti, ma anche al metodo generale usato in tutto il compendio di donne celebri, questo schema del capitolo della regina partenopea spicca per un motivo palese: oltre alla descrizione della protagonista

10 Fra parentesi quadre si fa riferimento alle suddivisioni del testo originale.

11 In certi casi le suddivisioni precedentemente citate sono state ulteriormente frammentate per rispondere in modo più efficiente a criteri contenutistici.

12 Data l'estensione della storia di Camiola, essa può essere successivamente suddivisa: a) [13-14] Liberazione di Rolando; b) [15-17a] Rolando infrange la promessa; c) [17b-30] Discorso di Camiola; d) [31-32] Reazione e conseguenze del discorso. 
non si include nessuna circostanza; detto in altri termini e come precedentemente avanzato, il capitolo CVI manca di una storia vera e propria, di un argomento da raccontare. Questo tratto contrasta con un segno d'identità che il $D M C$ può condividere con il De casibus, vale a dire, che "anticipando la codificazione rinascimentale, Boccaccio con le sue biografie non vuole solo descrivere con esattezza un uomo [o una donna] determinato e le sue azioni, ma cogliere in lui [o in lei] l'archetipo di una forma di vita" (Aricò 2015-2016: 235). La mancanza di eventi significativi narrati nel capitolo consacrato alla vita della sovrana partenopea fa sì che, di Giovanna, Boccaccio non possa fornire alcun "archetipo di [...] forma di vita", bensì una mera descrizione, dalla quale il lettore non è in grado di trarre alcun tipo di insegnamento.

Tuttavia, a nostro avviso, per capire questa anomalia si devono considerare diversi elementi. Come detto prima, Giovanna viveva ancora ai tempi della stesura del $D M C$. Infatti, sono proprio alcuni dei dati della sua biografia a permettere la datazione del capitolo. Più esattamente, il brano in cui Boccaccio afferma "Nam perpessa est intestina regulorum fratrum discordia et extera bella, non nunquam intra regni gremium debachata, sic et alieno crimine fugam exilium et coniugorum austeros mores"13 (1967: 446-448), dal quale Landau (1877: 211) inferì che il capitolo fu composto tra la fine del maggio e la metà del dicembre del 1362, periodo durante il quale la regina rimase vedova e, quindi, ebbe l'occasione di affrontare e superare le difficoltà narrate dal certaldese. Ciononostante -e come Ricci (1959: 17) e Zaccaria (1963: 293-294) dimostrarono-, l'aggiunta della biografia della regina non fu l'unica modifica che il $D M C$ sofferse verso l'estate del 1362 e, soprattutto, bisogna tener presente che certe particolarità della vita dell'autore che si manifestarono nella stessa data poterono determinare le suddette modifiche.

Sappiamo, grazie a una lettera che Boccaccio scrisse a Francesco Nelli, che lo scrittore si trovava in una precaria situazione economica in questo periodo e che, per cercare di porvi rimedio, Boccaccio aveva deciso di trasferirsi a Napoli nell'autunno di quello stesso 1362, dopo aver accettato la proposta di lavorare al servizio di Niccolò Acciauoli, Gran Siniscalco del Regno. Il certaldese, la cui stesura del $D M C$ era già a buon punto, potrebbe aver pensato di aggiungere qualche modifica per poter presentarla come dono ai suoi nuovi padroni a Napoli e come opera che elogiasse la sovrana partenopea. Per dirlo con le parole di Ricci (1959: 17-18),

Un dono di pregio ci voleva per testimoniare gratitudine ed ossequio. Ela necessità di far presto, per aver pronto il volume dopo pochi mesi, quando, passato il caldo estivo, sarebbe venuta l'ora di partire per Napoli, indusse il Boccaccio a concludere in fretta l'opera aggiungendovi pochi parti nuove [...]. Dato che l'opera doveva esser dedicata a [Andreina Acciaiuoli] una signora toscana residente nell'Italia meridionale, pensò infatti di sottolineare graziosamente questo particolare scrivendo la biografia di una Senese che aveva fatto risplendere a Messina le sue virtù; e apprestandosi a divenire suddito della regina Giovanna, non poteva certo esimersi dallo scrivere un

13 Ella infatti sopportò guerre all'interno, per la discordia tra i fratelli della famiglia reale, e al di fuori del Regno, talora infuriando nel cuore di esso; sopportò inoltre, per colpa altrui, la fugga, l'esilio, gli arcigni caratteri dei mariti. (Boccaccio 1967: 447-449). 
capitolo a lei dedicato.

Lasciando da parte le circostanze biografiche e lo scopo strumentale da parte dell'autore, bisogna ricordare che Giovanna nacque circa nell'anno 1327, per cui la monarca era poco più che trentenne quando diventò protagonista ultima del ciclo di biografie muliebri. Infatti, in un certo senso c'è da stupirsi se il capitolo non include nessuna vicenda notevole della regina, perché in realtà ve ne furono -e di grande rilievofino alla data della stesura del $D M C$. Basti pensare al suo difficile rapporto con il primo marito, Andrea d'Ungheria, all'assassinio di questo e, come conseguenza, all'invasione del Regno da parte del cognato, Luigi d'Ungheria, o al sofferto amore fin dagli anni della giovinezza con il cugino -e infine secondo marito- Luigi di Taranto ${ }^{14}$.

Per cercare di spiegare l'origine del particolare approccio alla vita di Giovanna un'altra sezione della raccolta boccaccesca diventa di importanza capitale: la dedica, anch'essa risalente alla stessa fase di stesura delle biografie di Camiola e della sovrana angioina.

Molto significativo per quanto riguarda entrambi questi fenomeni è l'avverbio con cui si apre l'opera: "pridie". Con questo "poco tempo addietro" Boccaccio indica che la stesura della dedica avvenne in una fase posteriore a quella della redazione - totale o parziale che sia - del DMC. In questa sezione, l'autore dedica la sua raccolta ad Andreina Acciaiuoli, allora contessa di Altavilla, ma -come egli stesso confessa- la nobildonna fiorentina non fu la prima dedicataria a cui pensò per il proprio lavoro:

ante alias venit in mentem ytalicum iubar illud prefulgidum ac singularis, non tantum feminarum, sed regum gloria, Iohanna, serenissima Ierusalem et Sicilie regina. Cuius pensatis, tam inclite prosapie et avorum fulgoribus, quam novis a se forti pectore quesitis laudibus, in desiderium mictendi illum humilem devotumque ante solium sue celsitudinis incidi ${ }^{15}$. (Boccaccio 1967: 18)

Ciononostante, dopo aver considerato che la sua opera fosse troppo rozza per una donna così importante, il certaldese conclude "nova indagine multis aliis perquisitis, ad extremum ab illustri regina in te [Andreina Acciaiuoli] votum deflexi meum; nec inmerito"16 (Boccaccio 1967: 18).

Dato che nelle accurate ricerche sulle fasi redazionali dell'opera nessuno degli studiosi prima citati hanno trovato traccia di una dedica anteriore a questa, non vi sono prove della verità dei motivi a cui l'autore allude, motivi che potrebbero anche rientrare nella retorica lusinghiera delle dediche. Così, lontano da sentirsi offesa per non essere

14 La guerra contro Carlo di Durazzo, nipote ed erede di Giovanna, come conseguenza delle differenze rispetto allo Scisma di Occidente, costituisce l'unico episodio notevole della vita della regina posteriore alla datazione del capitolo. Per il ruolo che Giovanna svolse nel Regno di Napoli, vedi De Feo (1969), Sabatini (1975), Angelillis (1977), Gleijeses e Gleijeses (1990).

15 Cercando la più degna, mi venne in mente, prima delle altre, quel fulgidissimo splendore d'Italia, gloria singolare, non solo delle donne, ma anche dei re, voglio dire Giovanna, gloriosissima regina di Gerusalemme e di Sicilia. Considerando, da un lato lo splendore della sua illustre prosapia e dei suoi avi, dall'altro le lodi nuove da lei meritate col suo forte spirito, venni nel proposito di presentare il mio modesto e devoto libretto al trono della Sua Altezza. (Boccaccio 1967: 19)

16 Dopo avere a lungo cercato molte altre a cui dedicare l'opera, volsi infine la mia scelta dalla regina a Te. E non senza ragione. (Boccaccio 1967: 19) 
stata la prima dedicataria della collezione, Andreina dovrebbe essere stata fiera di essere considerata, in un certo senso, come la seconda donna più illustre del Regno, e non avrebbe avuto torto. Per dirla con Kolsky, "[Boccaccio's] choice is based on the realization that real power in the Kingdom is in the hands of Niccolò Acciaiuoli to whom Boccaccio believed he had special rights of access" (2003: 115).

Ma cerchiamo di pensare ad altri motivi che abbiano potuto spingere Boccaccio al rifiuto dell'idea primigenia della dedica alla regina. Forse il certaldese avrebbe potuto considerare poco adeguato o insufficiente dedicare alla monarca un compendio di biografie attraverso le cui pagine sfilano egregie governanti di tutti i tempi, costringendola al ruolo di semplice spettatrice quando, invece, la stessa struttura dell'opera gli offriva la possibilità di farla diventare protagonista. E, fra l'altro, tenuto conto del criterio cronologico, occupando un posto di massimo rilievo all'interno del percorso.

Detto questo, sia tramite l'opera che nella dedica stessa, Andreina è esaltata, ma lo è di più Giovanna. Anzi, si potrebbe tracciare un parallelismo tra le parole con cui la regina viene descritta nella dedica e quelle con cui si chiude la sua biografia; se è già stato detto che nella prima sezione la monarca angioina è descritta come "ytalicum iubar illud prefulgidum ac singularis, non tantum feminarum, sed regum gloria" (Boccaccio 1967: 18), il capitolo CVI si chiude con l'affermazione dell'autore "quibus agentibus, ego non solum illam reor egregiam et splendida claritate conspicuam, sed singulare decus ytalicum, nullis hactenus nationibus simile visum"17 (Boccaccio 1967: 448).

Torniamo ancora una volta alle circostanze di Giovanna all'epoca della conclusione del $D M C$. Abbiamo già detto quanto sia eccezionale per questo tipo di raccolte includere la vita di un personaggio che sia ancora vivo, in un certo senso si può dire che questo modus operandi attenti alle regole costituenti del genere nel trattare, non più di personaggi, ma di persone. In effetti, alludendo al celebre episodio del Don Chisciotte, dove il cavaliere incontra Ginés de Pasamonte (I, 22), potremmo domandarci come mai la vita della regina partenopea sia inclusa in queste pagine quando i suoi giorni non erano ancora finiti.

La risposta a questa domanda sorge, tuttavia, chiara: mentre le centocinque biografie restanti presentano una struttura chiusa, l'ultima sezione è aperta. Infatti, il tono della narrazione è drasticamente diverso. Se Müller, paragonando il $D M C$ e La cité des dames di Christine de Pizan affermò che "Christine versteht ihr Buch nicht als historisches Werk, sondern als engagierte und subjektive Parteinahme zugunsten der Frauen" (1992: 151), questa osservazione non sarebbe valida come elemento divergente nei due cataloghi se ci limitiamo all'ultimo capitolo boccaccesco. È palese che le altre sezioni sono articolate secondo un voluto e sicuro principio storico che in non pochi casi risponde ad uno studio rigoroso delle fonti classiche e ad una riflessione profonda su di esse $^{18}$. In opposizione a ciò, la biografia di Giovanna è palesemente propagandistica, per niente imparziale e chiaramente soggettiva.

17 Perciò io la considero, non solo egregia e spettabile per lo splendore della fama, ma decoro singolare d'Italia, non mai visto finora da gente alcuna. (Boccaccio 1967: 449)

18 Si pensi ai capitoli che parlano di dee dell'Antichità, dove oltre allo studio mitologico e mitografico è evidente il forte influsso evemeristico, e alla ricerca quasi filologica che implica la biografia di Dido (XLII). 
In effetti, Boccaccio non fu il primo a parlare del regno di Giovanna, ma si può dire che le affermazioni contenute in $D M C$, CVI, (in modo particolare in 8-9 ${ }^{19}$ ), siano ben lungi dalle visioni che altri intellettuali dell'epoca diedero delle doti di governo e delle virtù personali della monarca angioina. Così, Petrarca, recatosi a Napoli nell'ottobre del 1343, descrisse Giovanna in una lettera a Barbato di Sulmona paragonandola ad una "lussuriosa Cleopatra" ${ }^{20} \mathrm{e}$ al contempo censurò il malgoverno e la corruzione della reggia partenopea identificandola con una nuova Babilonia ${ }^{21}$. Nello stesso modo, il primo volgarizzamento del $D M C$ in italiano offre una visione completamente diversa della sovrana napoletana. Questa versione in volgare, tradotta da Donato degli Albanzani seguendo un incarico di Niccolò d'Este, marchese di Ferrara, aggiorna la biografia finale raccontando i principali avvenimenti della vita di Giovanna fino alla sua morte e, soprattutto, fornendo un punto di vista molto più obiettivo e sprovvisto dal valore celebrativo e propagandistico dell'opera di Boccaccio. In effetti, lo stesso Albanzani indicò che il certaldese "studiosamente adornò [Giovanna] di maravigliosi lodi, perché quest'opera pervenisse alle sue mani” (Boccaccio 1881: 390)22.

19 "Quod cum permaximum sit dominium nec id sit a mulieribus possideri consuetum, non minus miraculi quam claritatis affert, si satis inspicimus. Et, quod longe mirabilius est, sufficit illi ad imperium animus: tam perlucidam adhuc avorum indolem servat. Ea enim, postquam regio dyademate insignita est, virtute insurgens valida, adeo purgavit, nedum civitates et domestica loca, verum Alpes, saltus devios, nemora et ferarum lustra scelesta hominum manu, ut aufugeret omnis terrefacta aut se celsis clauderet arcibus; quos, agmine armatorum emisso sub egregio duce, non ante locorum talium obsidionem solveret, quam, captis munitionibus, infandos homines affecisset supplicio, quod precedentium regum aliquis aut noluit aut fecisse nequivit; eoque redegit terras quas possidet, ut non solum inops, sed et opulentus cantando nocte dieque possit quo velit tutus iter arripere; et - quod non minus salubre - insignes viros Regnique proceres tanta frenavit modestia et eorum mores solutos retraxit in melius, ut, posita superbia veteri, qui reges olim parvipendebant, hodie faciem irate mulieris horrescant”. (Boccaccio 1967: 446)

["E poiché signorie grandi come questa non sono di solito possedute da donne, quella di Giovanna non offre - a veder bene - minor ragione di meraviglia che di fama.

Inoltre è motivo di anche maggior ammirazione il fatto che Giovanna ha l'animo all'altezza del suo governo, ella che serba ancor così pura l'indole dei suoi antenati.

Poiché fu insignita della corona regale, validamente ergendosi colla sua virtù, liberò non solo la città e i luoghi abitati, ma anche le montagne e le pendici impervie, i boschi e i covili delle fiere dalla presenza di bande di uomini scellerati. Esse fuggirono spaventate e si rinchiusero nelle loro alte rocche; ma la regina mandò contro loro un esercito agli ordini di un valoroso comandante e non cessò di stringerle d'assedio prima di aver fatto saltare le difese e di aver mandato al supplizio quegli uomini scellerati.

Ella compì dunque un'impresa che i re precedenti o non avevano voluto o non avevano potuto eseguire. Le terre che ora possiede, Giovanna ha ordinato in modo che chiunque voglia passar per esse - sia povero o ricco può farlo con sicurezza notte e giorno, cantando.

Inoltre (cosa non meno salutare) frenò con tanta modestia gli uomini insigni e i principi del regno, traendo al meglio i loro rilassati costumi, che oggi, deposta l'antica superbia, temono l'aspetto della regina, quando s'adira, gli stessi che un tempo disprezzavano i re"]. (Boccaccio 1967: 447)

20 Vedi Familiare V, III, 1. Per più informazioni sull'immagine di Giovanna fra gli intellettuali del Trecento rimandiamo a Lokaj (2000) e Caputo (2008: 131-147).

21 Nella Familiare V, III, 8, in seguito ad un incontro presso la corte partenopea, Petrarca scrisse

"Reginas adii et reginarum consilio interfui. Proh pudor, quale monstrum! Auferat ad italico celo Deus genus hoc pestis! Putabam Cristum apud Memphim et Babilonem Mechamque contemni; compatior tibi, mea nobilis Parthenope; vere tu harum quelibet facta es; nulla pietas, nulla veritas, nulla fides"

["Sono andato dalle regine e ho assistito al consiglio delle regine. Che vergogna, che mostruosità! Dio porti via dal cielo d'Italia questo genere di peste! Pensavo che Cristo venisse disprezzato presso Menfi, Babilonia e la Mecca. Ti compatisco, o mia nobile Partenope, tu che sei veramente diventata una di quelle città: nessuna pietà, né verità, né fede"].

22 Come Kolsky affermò, da una prospettiva prettamente storica, il risultato dell'atteggiamento boccaccesco nei confronti della vita della sovrana "was a falsification of the truth -a distortion which the translator aimed to correct, from an apparently disinterested perspective, by proceeding with an 'objective' historical narrative” (2003: 173). 
Gli ornamenti a cui il traduttore fa riferimento hanno a che vedere essenzialmente con il mondo politico partenopeo descritto dal certaldese nella vita della monarca (vedi nota 19). Nonostante Boccaccio insista sul fatto che Giovanna abbia "l'animo all'altezza del suo governo" (1967: 447), altre fonti - fra le quali Petrarca e Albanzani - sono del parere che sia l'animo sia le doti di governo della sovrana e, soprattutto, il suo controllo sulla corruzione della corte napoletana fossero ben distanti dal poter descriversi con le eccelse parole che il certaldese adopera. A confermare che il giudizio boccaccesco ha uno scopo propagandistico e non cerca altro che apportare benefici personali all'autore, sta il fatto che, pochissimo tempo dopo la composizione dell'opera, quando essa fu tradotta da Albanzani in un ambiente - quello di Ferrara - che poco aveva a che fare con la corte angioina, la vita della regina perde completamente il tono assunto nel $D M C$ latino, e lo fa in modo così drastico da far sì che la protagonista quasi divenga un esempio di donna che non dovrebbe essere imitata.

Per concludere e in vista di questi motivi, a nostro parere, il capitolo CVI del DMC potrebbe essere considerato, se non come la vera e propria dedica della raccolta, almeno come un capitolo in cui l'intenzionalità di rendere omaggio ad una persona tipica delle dediche è presente con intenso vigore. Nell'ammettere che la primigenia dedicataria della silloge era stata Giovanna e non Andreina, Boccaccio cade in quel meccanismo di soppressione e aggiunta ulteriore della dedica che Genette definì come un" "opération sans doute plus rare que la suppression simple, puisqu'elle aggrave l'abandon d'une infidélité positive" (1987: 131). Tuttavia, nella silloge boccaccesca non è lecito parlare di abbandono, perché Giovanna non sparisce dall'opera, anzi, passa ad occupare il posto più privilegiato a cui possa aspirare: la chiusura, il punto omega di un percorso iniziato con la prima delle donne, Eva.

Ciononostante, come già notato, la sovrana non diviene protagonista di un capitolo nello stesso modo né secondo gli stessi principi del resto delle donne celebri che sfilano lungo la raccolta. La sua vita è modellata dall'autore con uno scopo strumentale che risponde, ancora una volta, alle principali intenzionalità della dedica; in questo caso, alla "fonction directement économique [et/ou à] son rôle de patronage" (Genette 1987: 138). Tuttavia, queste funzioni vengono esercitate non da una posizione liminare -per alludere alle soglie genettiane-, ma dallo stesso cuore dell'opera, con delle sfumature non solo fortemente intratestuali, dati i rapporti del capitolo con la dedica vera e propria, bensì anche chiaramente intradiegetiche. Vale a dire che, seguendo un'innovativa strategia di costruzione testuale, l'autore modella il $D M C$ come un'opera tramite la quale si celebrano le virtù sia della sorella del Gran Siniscalco, sia della regina, in modo che, se il viaggio a Napoli non fosse stato troncato, Boccaccio avrebbe potuto accontentare entrambe le donne con una silloge che, al di là di questo valore strumentale immediato, ha continuato ad offrire spunti per la riflessione critica e momenti di piacere letterario lungo i secoli.

\section{BIBLIOGRAFIA}

ANGELILLIS, Ciro (1977): Nuove luci sulle vicende della regina Giovanna I di Napoli. Monte Sant'Angelo: Centro Studi Micaelici e Garganici.

ARICÒ, Denise (2015-2016): "Per le antiche ruine con nuove scritture'. Le biografie politiche nel De casibus di Boccaccio", Heliotropia. 12-13: 233-261. 
BILLANOVICH, Giuseppe (1953): "Il Petrarca, il Boccaccio, Zanobi da Strada e le tradizioni dei testi della 'Cronaca' di Ugo Falcando e di alcune 'Vite' di Pontefici”, Rinascimento. IV: 17-26.

BOCCACCIO, Giovanni (1881): Delle donne famose, tr. Donato degli Albanzani. Bologna: Commissione pei testi di lingua.

(1951): De las ilustres mujeres en romance (Zaragoza, 1494). Madrid: Real Academia Española.

(1967): De mulieribus claris, ed. Vittorio Zaccaria. Milano: Mondadori.

BRANCA, Vittore; ZACCARIA, Vittorio (1996): "Un altro codice del De mulieribus claris del Boccaccio", Studi sul Boccaccio. 24: 3-6.

CAPUTO, Vincenzo (2008): "Una galleria di donne illustri: il De mulieribus claris di Giovanni Boccaccio", Cahiers d'études italiennes. 8: 131-147.

DE FEO, Italo (1969): Giovanna d'Angiò. Napoli: Fausto Fiorentino.

DÍAZ-CORRALEJO, Violeta (2001): "La traducción castellana del De mulieribus claris", Cuadernos de filología italiana. Número extraordinario: 241-261.

FERNÁNDEZ MURGA, Félix; PASCUAL RODRÍGUEZ, José Antonio (1975): "La traducción española del De mulieribus claris de Boccaccio", Filología moderna. LV: 499-511.

(1977): "Anotaciones sobre la traducción española del De mulieribus claris de Boccaccio", Studia philologica salmanticensia. 1: 53-64.

FILOSA, Elsa (2012): Tre studi sul "De mulieribus claris". Milano: LED.

GENETTE, Gérard (1987): Seuils. Paris: Éditions du Seuils.

GIROLAMO (1999): Gli uomini illustri. Roma: EDB.

GLEIJESES, Vittorio; GLEIJESES, Lidia (1990): La regina Giovanna d' Angiò. Napoli: Tommaso Marotta editore.

HORTIS, Attilio (1877): Le donne famose descritte da Giovanni Boccaccio. Trieste: Stabilimento art. Tip. G. Caprin.

KOLSKY, Stephen D. (2003): The Genealogy of Women. Studies in Boccaccio's "De mulieribus claris". New York: Peter Lang.

LANDAU, Marcus (1877): Giovanni Boccaccio: Sein Leben und Seine Werke. Stuttgart: Cotta.

LOKAJ, Rodnej J. (2000): "La Cleopatra napoletana: Giovanna d'Angiò nelle Familiares di Petrarca", Giornale storico della letteratura italiana. 117: 481-521.

MORABITO, Raffaele (1988): "La diffusione della storia di Griselda dal XIV al XX secolo", Studi sul Boccaccio. XVII: 237-285.

(1990): La storia di Griselda in Europa. Roma: Japadre.

(1993): Una sacra rappresentazione profana. Fortune di Griselda nel Quattrocento italiano. Tübingen: Max Niemeyer.

MÜLLER, Ricarda (1992): Ein Frauenbuch des frühen Humanismus. Untersuchungen zu Boccaccios 'De mulieribus claris'. Stuttgart: Franz Steiner Verlag.

NARDONNE, Jean-Luc (2000): L'histoire de Griselda. Tome I: prose et poésie. Toulouse: Presses Universitaires du Mirail. 
(2001): L'histoire de Griselda. Tome II: théâtre. Toulouse: Presses Universitaires du Mirail.

NUVOLONI, Laura (2003): "De mulieribus claris: un frammento”, Studi sul Boccaccio. 31: 23-26.

PETRARCA, Francesco (2007-2012): De viris illustribus. 3 vol. Firenze: Lettere.

RICCI, Pier Giorgio (1959): "Studi sulle opere latine e volgari del Boccaccio", Rinascimento. X, I: 1-21.

RICO, Francisco (2002): El sueño del humanismo. De Petrarca a Erasmo. Barcelona: Destino.

(2013): Ritratti allo specchio (Boccaccio, Petrarca). Padova: Antenore.

RODRÍGUEZ MESA, Francisco José (2009): "Von etlichen Frowen: una modificación de Steinhöwel sobre la obra de Boccaccio", Estudios filológicos alemanes. 18: 127-138.

ROUSH, Sherry (2015): Speaking Spirits. Ventriloquizing the Death in Renaissance. Toronto: University of Toronto Press.

SABATINI, Francesco (1975): Napoli angioina: cultura e società. Napoli: Edizioni scientifiche italiane.

TORRETTA, Laura (1902a): "Il 'Liber de claris mulieribus' di Giovanni Boccaccio". Giornale storico della letteratura italiana. 39: 252-292.

(1902b): "Il 'Liber de claris mulieribus' di Giovanni Boccaccio". Giornale storico della letteratura italiana. 40: 35-65.

ZACCARIA, Vittorio (1963): "Le fasi redazionali del De mulieribus claris", Studi sul Boccaccio. I: 252-332.

ZAPPACOSTA, Guglielmo (1973): "Per il testo del De mulieribus claris. I. Il Cod. Laur. Pluteo XC sup. 98 e il testo dell'opera", Studi sul Boccaccio. 7: 239-245.

\section{PERFIL ACADÉMICO-PROFESIONAL}

Profesor Ayudante Doctor de Filología Italiana en la Universidad de Córdoba. Su investigación se ha centrado en diversos aspectos de la literatura italiana medieval y renacentista, como el análisis de la lírica petrarquista y su difusión, en particular en el sur de la península italiana durante el Quattrocento (Giannantonio de Petruciis, Rustico Romano, el «Cansonero» del conde de Popoli), o Boccaccio y la tipología de las formas narrativas breves. Asimismo, se ha ocupado del papel de la mujer en algunas obras italianas del Trecento, principalmente en Boccaccio (Decameron, De mulieribus claris) y Petrarca (De insigni obedientia et fide uxoria, Rerum vulgarium fragmenta).

En los últimos años, ha impartido diversos seminarios en calidad de profesor visitante en varias universidades extranjeras, como la Università La Sapienza di Roma, la Università degli Studi di Udine o la Universidad Nacional Autónoma de México, y ha sido nombrado Investigador Asociado de la Biblioteca Nacional de Uruguay.

Fecha de recepción: 08/05/2019

Fecha de aceptación: 22/07/2019 